\title{
Yeast Two-Hybrid Assay Reveals Several Interactors of EphB6 Receptor
}

\author{
Brian P. Fox ${ }^{1,2}$ and Raj P. Kandpal ${ }^{1, *}$ \\ ${ }^{I}$ Department of Basic Medical Sciences, Western University of Health Sciences, Pomona, CA; ${ }^{2}$ Department of Biologi- \\ cal Sciences, Fordham University, Bronx, NY, USA
}

\begin{abstract}
EphB6, a kinase-deficient receptor, belongs to the Eph family of receptor tyrosine kinases. It can participate in active cell-to-cell signaling pathways following dimerization with and phosphorylation by EphB1 and possibly other members of the EphB family. EphB6 is a positive prognostic marker in neuroblastoma. The loss of EphB6 expression in melanomas, non-small cell lung carcinomas and breast cancer appears to be associated with a more advanced stage of these cancers. Despite the apparent role for EphB6 in preventing the progression of these cancers, little is known about EphB6-mediated signaling in cancer. We have identified 16 unique proteins that are capable of interacting with the cytoplasmic domain of EphB6 in the yeast two-hybrid assay. Furthermore, the interaction of a subset of these proteins (aldolaseA, dynactin, clusterin, TMEM25 and plekstrin homology domain-containing family B member 1) with EphB6 in mammalian cells was confirmed by employing a co-immunoprecipitation strategy. The identification of these interacting proteins suggests pathways mediated by EphB6.
\end{abstract}

\section{INTRODUCTION}

The Eph receptors are the largest family of receptor tyrosine kinases (RTK) in humans with fourteen members identified to date [1]. These receptors are divided into A and B classes based on their homology to one another as well as their affinity for their ephrin ligands. Following the binding of an ephrin ligand to an Eph receptor, signals are transmitted into the ligand-expressing cell as well as the receptorexpressing cell. These signaling events are known to be involved in normal animal development and in the progression of various cancers. Specifically, Eph and ephrin signaling has been well characterized in the developing nervous system and appears to be mediated by integrin activation and actin cytoskeletal rearrangements [2-7].

EphB6 is lacking intrinsic tyrosine kinase activity as a result of several changes in the amino acid sequence of the conserved kinase domain [8]. Despite these alterations, EphB6 has been shown to promote protein tyrosine phosphorylation in Jurkat cells following cross-linking of EphB6 and CD3 [9]. The ability of EphB6 to promote protein phosphorylation can at least in part be explained by a study showing that EphB6 undergoes transphosphorylation when heteromerized with EphB1 [10]. Interestingly, EphB6 is capable of positively and negatively regulating cell adhesion as well as migration in two mammalian cell lines following stimulation with ephrinB2 [11]. The differential response is dependant on the concentration of the ligand used for stimulation and is correlated to Src-dependant phosphorylation of the cytoplasmic domain of EphB6 [11]. Together these studies show that EphB6 is capable of altering cellular phenotypes that are known to be involved in the progression of cancer.

*Address correspondence to this author at the Department of Basic Medical Sciences, Western University of Health Sciences, 309 E. Second Street, Pomona, CA 91766, USA; E-mail: rkandpal@westernu.edu
The expression of EphB6 appears to correlate with the treatment outcome of certain cancers. In metastatic melanoma, for instance, EphB6 expression is significantly less than in non-metastatic melanoma or normal melanocytes [12]. Also, it has been shown that in non-small cell lung carcinoma, patients with tumors lacking the EphB6 transcript were three times more likely to develop metastases than those patients with EphB6-expressing tumors [13]. Furthermore, strong evidence exists for the utility of EphB6 expression as a positive prognostic indicator in neuroblastoma [1416]. We have shown that the levels of EphB6 transcript are significantly decreased in the most aggressive breast cancer cell lines [17].

The yeast two-hybrid assay is a powerful technique to identify protein-protein interactions $[18,19]$. The identification of interacting partners may allow placement of a protein into specific intracellular pathways and can help elucidate the cellular mechanisms for observed phenotypic alterations mediated by a specific protein $[18,19]$. This methodology has allowed us to identify sixteen proteins that interact with the cytoplasmic domain of EphB6 in yeast cells. We selected five interactors and demonstrated that these proteins are capable of interacting with EphB6 in mammalian cells. We have used these interacting proteins as a basis to discuss EphB6-mediated signaling in tumor cells.

\section{MATERIALS AND METHODS}

\section{Generation of EphB6 Bait Construct}

The cytoplasmic domain of EphB6 (amino acids 6021006 of NCBI database entry NP_004436.1) was amplified from a full length EphB6 construct using the primers 5'GATGAAGAATTCGTCGTCTTCCAGCGGAAGCGGC GTG3' and 5'GATCAGGATCCGACCTCCACTGAGCCCT GCTG3' corresponding to bases 2636-2660 and 3850-3830 
of NCBI entry NM 004445, respectively. The amplified product was digested with EcoRI and BamHI and cloned into the yeast vector pGBKT7. The clone was sequenced to confirm that the EphB6 insert was in frame with the Ga14 DNA binding-domain (DNA-BD) of the vector.

\section{Yeast Two-Hybrid Screening}

A human brain cDNA library made in the yeast fusion vector pGADT7-Rec containing the Gal4 activation domain (AD) was used to isolate EphB6 interactors. The EphB6DNA-BD construct was transformed into AH109 yeast cells using the Yeastmaker Yeast Transformation System 2 (Clontech) to generate a transformant expressing the EphB6-BD fusion protein. A liquid culture of this transformant was mated to Y187 yeast cells that had been pretransformed with a human brain cDNA library (Clontech). After mating, the yeast culture was plated onto a synthetic agar based medium that lacked tryptophan, leucine and histidine. This synthetic medium, designated as triple dropout (TDO), is used to select for those yeast cells that show activation of His 3 gene transcription due to the presence of both EphB6-DNA-BD and pGADT7-Rec plasmids. The transcription of His3 gene is under the control of the Gal4 protein and its activation indicates the juxtaposition of the Gal4-DNA binding domain and the transcription activation domain. The yeast colonies that survived on TDO medium were subsequently streaked onto quadruple dropout (QDO) plates. The QDO medium contained the chromogenic substrate 5-Bromo-4-Chloro-3indolyl a-D-galactopyranoside (X- $\alpha-\mathrm{Gal}, \mathrm{Clontech})$ and lacked tryptophan, leucine, histidine and adenine. The bluecolored colonies from the QDO plates were grown individually in liquid cultures and plasmid DNA was isolated from these cells using the Zymoprep yeast plasmid miniprep kit (Zymo Research). The plasmid DNA was used to transform E.coli and the transformation mixture plated onto LB plates in the presence of ampicillin to select for colonies that have the pGADT7-Rec vector containing the cDNA insert of interest. The plasmids from these colonies were isolated and sequenced. The sequences were analyzed by BLAST on NCBI databases.

\section{Cloning of Selected Genes into Tagged Vectors for Co- Immunoprecipitation Analysis}

Aldolase A, clusterin, dynactin, plekstrin homology domain-containing family B member 1 and TMEM 25 were cloned into the pCDNA4 TOmycHis A vector (Invitrogen). EphB6 was cloned into the pCDNA3.1V5His vector (Invitrogen). The cloning sites, primers and templates used to amplify these transcripts are listed in Table 1. The clones were sequenced to confirm that the gene coding sequences are in frame with the His or V5 tag present on the vector.

\section{Generation of Stable Clones in MDA-MB-231 Cells}

A full length EphB6 construct or pCDNA3.1+ (Invitrogen) was used to transfect MDA-MB-231 cells using Lipofectamine 2000 (Invitrogen) according to the manufacturer's recommendations. Prior to transfection these cells were grown as described previously [17]. Following transfection, the cells were grown in media containing $500 \mu \mathrm{g} / \mathrm{ml}$ G418, and resistant clones were isolated, expanded and analyzed for EphB6 transcript and protein levels.

\section{Analysis of EphB6 Transcript and Protein Levels in MDA-MB-231 Stable Clones}

\section{Semi-Quantitative RT-PCR}

The QIAGEN One Step RT-PCR kit was used for all reactions. Equal amounts of RNA from each cell line, as confirmed by amplification of actin transcript, were used in parallel reactions. The reaction mixtures $(10 \mathrm{ml})$ containing 200 ng DNase-treated RNA, 6.0 pmol each of forward and reverse primers, $4.0 \mathrm{nmol}$ dNTPs, $0.4 \mathrm{ml}$ of a mixture of reverse transcriptase and Taq DNA polymerase and $1 \mathrm{X}$ supplied reaction buffer were subjected to RT-PCR in a Perkin Elmer 9600 Gene Amp PCR System. The reverse transcription reaction was performed at $50^{\circ} \mathrm{C}$ for 30 minutes, fol-

Table 1. Cloning Strategy For Generating Full Length Constructs

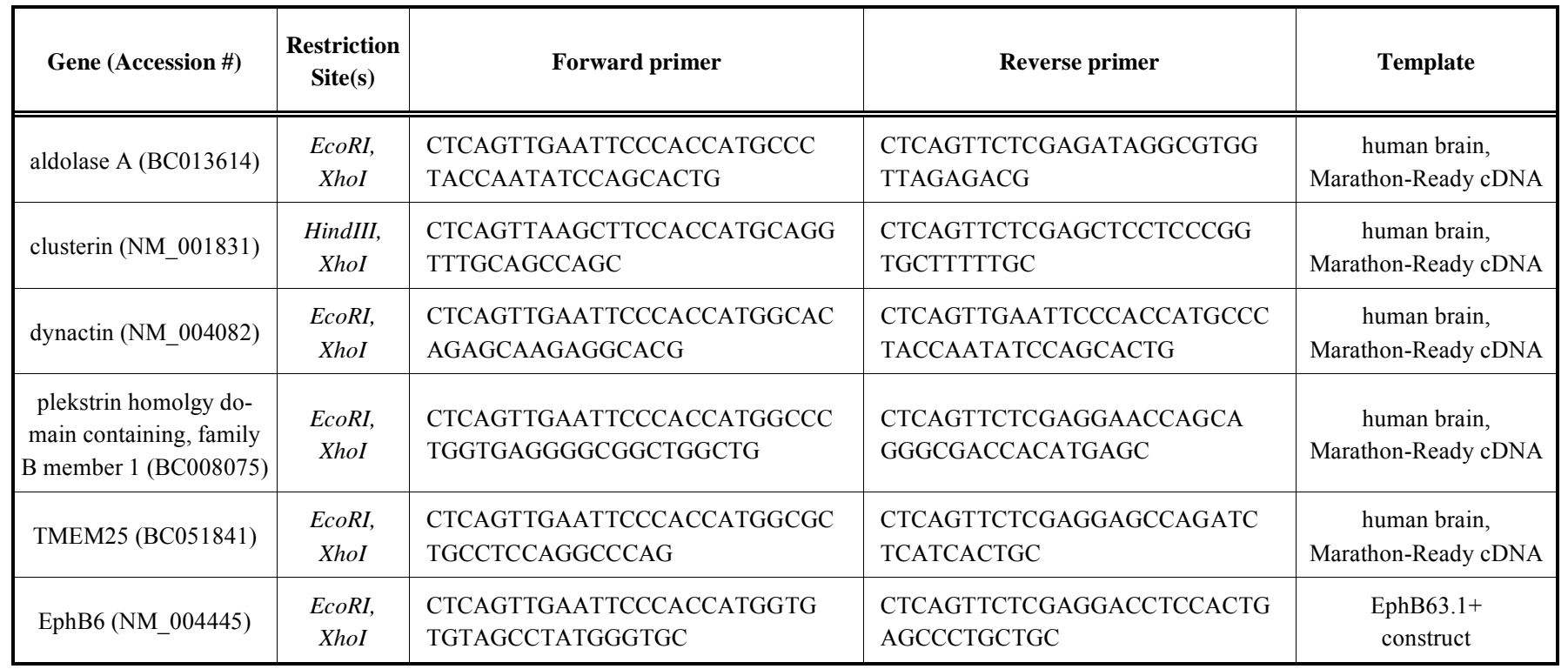


lowed by a 15 minute incubation at $95^{\circ} \mathrm{C}$ to activate the HotStarTaq ${ }^{\mathrm{TM}}$ DNA polymerase. Each PCR cycle consisted of the following incubations: $94^{\circ} \mathrm{C}$ for 30 seconds, $58^{\circ} \mathrm{C}$ for 30 seconds, and $72^{\circ} \mathrm{C}$ for $1 \mathrm{~min}$. The sequences of forward primer and reverse primer for EphB6 (5'GTTCTGGACGA CCAGCGACG3' and 5'GACGTTCAGTTGCAGTCCAG3') correspond to bases $968-987$ and $1375-1356$ of the NCBI database entry NM_004445, respectively. The forward and reverse primer sequences for actin (5'CTGACTGACTACCT CATGAAG 3' and 5' ATCCACATCTGCTGGAAGGTG3') correspond to bases 626-646 and 1143-1123 of the NCBI database entry NM_001101, respectively.

\section{Western Blotting}

The cells were grown to the logarithmic phase in appropriate media in $10 \mathrm{~cm}^{2}$ plates as described above. The medium was removed and the plates were washed with ice-cold PBS. An aliquot $(500 \mu 1)$ of cell lysis buffer (Cell Signaling Technology) containing $1 \mathrm{mM}$ phenylmethylsulphonyl fluoride (Sigma) was added to each plate and the plates were kept on ice for 5 minutes. The cell lysate was collected in a $1.5 \mathrm{ml}$ tube. The lysates were sonicated 4 times for 5 seconds each using the Misonix 3000 sonicator at a setting of 2.0 and the homogenate centrifuged at $16,000 \mathrm{x} \mathrm{g}$ in a microfuge at $4^{\circ} \mathrm{C}$ for 10 minutes. The supernatant was collected in a fresh tube and used for analysis. Approximately $50 \mu \mathrm{g}$ of protein (in a volume of $20 \mu \mathrm{l}$ ), as determined by the Bradford reagent (Sigma), was combined with $10 \mu \mathrm{l}$ of $3 \mathrm{x}$ loading buffer (Cell Signaling Technology) supplemented with $125 \mathrm{mM}$ dithiothreitol. The samples were loaded onto a $10 \%$ SDSpolyacrylamide gel and electrophoresed at $60 \mathrm{~mA}$ for approximately 1.5 hours. The proteins were transferred from the gel to a PVDF membrane (Amersham Biosciences) and processed as described below for the detection of EphB6 or $\beta$-actin.

The membrane was incubated for one hour at room temperature with blocking buffer that included Tris buffered saline (TBS) and 5\% nonfat dry milk. The membrane was subsequently incubated with $5 \mu \mathrm{g}$ of goat anti-mouse EphB6 polyclonal antibody (R\&D systems) in $15 \mathrm{ml}$ of TBS containing $0.1 \%$ Tween $\AA 20$ (TBS-T) and 5\% bovine serum albumin (BSA) at $4{ }^{\circ} \mathrm{C}$ overnight. The blot was then washed 3 times for 5 minutes each with TBS-T at room temperature and then incubated with an HRP-conjugated donkey antigoat-antibody (Santa Cruz Biotechnology) at a dilution of 1:100000 in TBS-T for one hour at room temperature. The membrane was washed 3 times for 5 minutes each with TBS$\mathrm{T}$ and incubated with HRP substrate (Millipore). The addition of the substrate generated a chemiluminescent signal that was detected following exposure to an X-ray film, and the intensity of this signal was used to determine the relative abundance of the EphB6 protein.

To determine the relative amounts of total protein loaded for each sample, the membrane was washed to remove the bound antibodies as per the membrane manufacturer's recommendations and re-probed with a monoclonal mouse antibody to human $\beta$-actin (Sigma). First, the membrane was incubated in blocking buffer for one hour at room temperature and then incubated with the anti- $\beta$-actin antibody, at a dilution of 1:3000, in blocking buffer for one hour at room temperature. The membrane was then washed three times for 5 minutes each with TBS-T and incubated with HRP- conjugated goat anti-mouse antibody (Santa Cruz Biotechnology) at a dilution of 1:10000 in blocking buffer for one hour at room temperature. Next, the membrane was washed three times for 5 minutes each with TBS-T and incubated with the HRP substrate (Millipore). Finally, the membrane was exposed to an X-ray film to determine the abundance of $\beta$-actin protein.

\section{Transfection of EphB6 Interactor Constructs into Mam- malian Cells for Co-Immunoprecipitation}

The constructs coding for the interacting proteins identified by the yeast two- hybrid assay were transfected into HEK293T cells or MDA-MB-231 stable clones using Lipofectamine 2000 (Invitrogen). Thirty hours after transfection, media were removed, plates were washed with ice cold PBS and cell lysates were prepared using $1 \mathrm{X}$ lysis buffer (Cell Signaling Technology) containing $1 \mathrm{mM}$ phenylmethylsulphonyl fluoride. The lysate $(20 \mu \mathrm{l})$ was combined with $10 \mu \mathrm{l}$ of $3 \mathrm{X}$ loading buffer (Cell Signaling Technology) supplemented with $125 \mathrm{mM}$ DTT. To detect the presence of the fusion proteins, the samples were electrophoresed, the proteins were transferred to a PVDF membrane (Amersham Biosciences) and processed for detection of the myc epitope.

Immunoprecipitation was performed by overnight incubation of lysates $(200 \mu \mathrm{l})$ from transfected cells with $2 \mu \mathrm{g}$ anti-EphB6 antibody (R\&D systems) with gentle rocking at $4^{\circ} \mathrm{C}$. A suspension of $20 \mu \mathrm{l}$ protein $\mathrm{G}$ plus-agarose beads (Santa Cruz Biotechnology) was added to the above mixture and the mixtures were incubated for 3 hours at $4{ }^{\circ} \mathrm{C}$ with gentle rocking. The samples were spun at $16,000 \mathrm{x} g$ for $30 \mathrm{sec}-$ onds at $4{ }^{\circ} \mathrm{C}$ in a microfuge and the supernatant discarded. The pellet containing the agarose beads was suspended in $1 \mathrm{X}$ lysis buffer (Cell Signaling Technology) and spun at 16,000 $\mathrm{x} \mathrm{g}$ in a microfuge for 30 seconds at $4^{\circ} \mathrm{C}$. This process was repeated four times. The beads were then mixed with $30 \mu \mathrm{l}$ of $3 \mathrm{X}$ loading buffer (Cell Signaling Technology) supplemented with $125 \mathrm{mM}$ dithioreitol. The mixture was heated at $100^{\circ} \mathrm{C}$ for 5 minutes and a $25 \mu \mathrm{l}$ aliquot was loaded onto a $10 \%$ SDS-polyacrylamide gel and processed for detection of the myc epiptope.

\section{RESULTS}

In order to identify proteins interacting with the cytoplasmic domain of human EphB6 using the yeast two-hybrid assay, a sequence coding for amino acids 602-1006 of NCBI database entry NP_004436.1 was cloned into the yeast vector pGBKT7 (Invitrogen). The EphB6 cytoplasmic region has a tyrosine kinase (TK) domain, a sterile-alpha-motif (SAM) domain and a postsynaptic density, zona-occludens (PDZ) domain [20]. The cloning strategy is described in the materials and methods section and the identity and relative position of the domains present within this portion of EphB6 are shown in Fig. (1). Sequencing of a clone revealed inframe fusion of the cytoplasmic domain of EphB6 with the Gal4 DNA binding domain, and the clone was named EphB6-DNA-BD. The expression of the fusion protein in AH109 cells transformed with EphB6-DNA-BD construct was confirmed by Western blotting using an antibody against the c-myc epitope present on the vector (data not shown). The transformed AH109 yeast cells were mated with Y187 yeast cells that had been transformed with a human brain 


\section{pGBKT7 domains $\quad$ EphB6 domains}

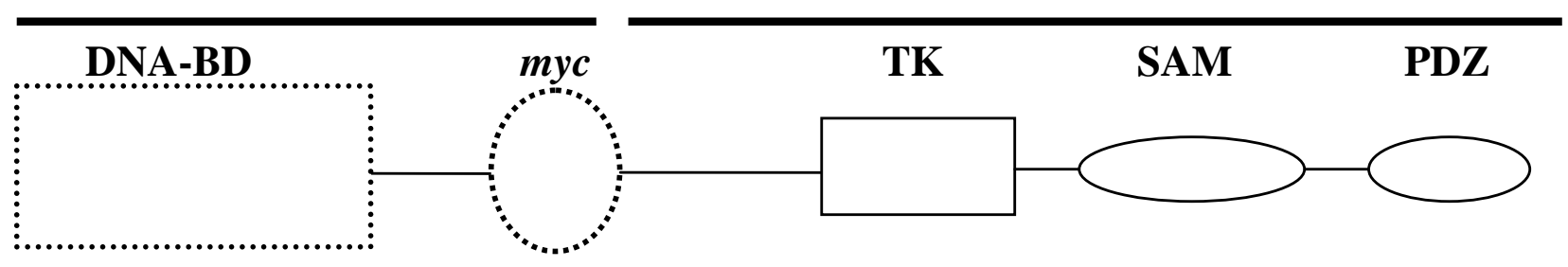

Fig. (1). Illustration of the domains present with the EphB6-DNA-BD clone used for yeast two hybrid analysis. DNA-BD = Gal4 DNA binding domain; $\mathrm{TK}=$ tyrosine kinase; $\mathrm{SAM}=$ Sterile alpha motif; $\mathrm{PDZ}=$ post-synaptic density, zona-occludens .

cDNA library (Clontech). This library was cloned into a yeast vector containing the GAL4 activation domain (AD). The mated yeast culture yielded 140 colonies when plated on triple dropout (TDO) agar growth medium lacking leucine, tryptophan and histidine. These colonies were further selected by re-streaking them onto agar plates of quadruple dropout (QDO) medium lacking adenine, leucine, tryptophan and histidine. The QDO medium also contained 5-Bromo-4Chloro-3-indolyl a-D-galactopyranoside (X- $\alpha-\mathrm{Gal})$. The juxtaposition of interacting proteins and consequent activation of $\alpha$-galactosidase (MELI) gene transcription led to the production of dark blue color in 32 yeast colonies. The sequences of cDNAs harbored by these yeast colonies, when analyzed by BLAST, matched to16 unique entries in the database (Table 2). These cDNAs included a mitochondrial membrane-bound protein (NADH dehydrogenase), 3 plasma membrane-bound proteins (fibroblast growth factor receptor substrate 2, transmembrane protein 25 and glycoprotein M6B) and 12 cytosolic proteins. The potential interactions between EphB6 and several of the proteins listed in Table 2 (dynactin 1, clusterin, transmembrane protein 25 -TMEM25, aldolase $\mathrm{A}$, and plekstrin homology domain-containing family $B$ member 1) were investigated in mammalian cells.

To perform the above analyses, full length cDNAs of transmembrane protein 25 (TMEM25), dynactin 1, clusterin, plekstrin homology domain-containing family B member 1 and aldolase $\mathrm{A}$ were cloned into the mammalian expression vector pCDNA4TOmycHis (Invitrogen) containing his and $m y c$ epitopes. Full length EphB6 was cloned into the mammalian expression vector pCDNA3.1V5His (Invitrogen) containing his and V5 epitopes. The candidate interacting protein constructs were individually transfected into HEK293T cells along with the EphB6 V5-His construct. A blot of cell extracts probed with anti-myc antibody confirmed the presence of fusion proteins in transfected cells based on their expected molecular weights (Fig. 2A). Similarly, the presence of EphB6 in the transfected cells was confirmed by

Table 2. Proteins Interacting with the Cytoplasmic Domain of EphB6

\begin{tabular}{|c|c|}
\hline NM_005001.1 & NADH dehydrogenase (ubiquinone) 1 alpha subcomplex (NDUFA7) \\
\hline NM_006654.2 & Fibroblast growth factor receptor substrate 2 (FRS2) \\
\hline NM_000034.2 & Aldolase A, fructose-bisphosphate (ALDOA) \\
\hline NM_002567.2 & Phosphatidylethanolamine binding protein 1 (PEBP1) \\
\hline NM_004082.2 & Dynactin 1 (p150, glued homolog, Drosophila) \\
\hline NM_001831.2 & Clusterin (CLU) \\
\hline NM_005439.1 & Myeloid leukemia factor 2 (MLF2) \\
\hline NM_173614.2 & NODAL modulator 2 (NOMO2) \\
\hline NM_033213.2 & Zinc finger protein $670(\mathrm{ZNF670})$ \\
\hline NM_021200.1 & Plekstrin homology domain containing, family B (evectins) member 1 (PLEKHB1) \\
\hline
\end{tabular}


A.

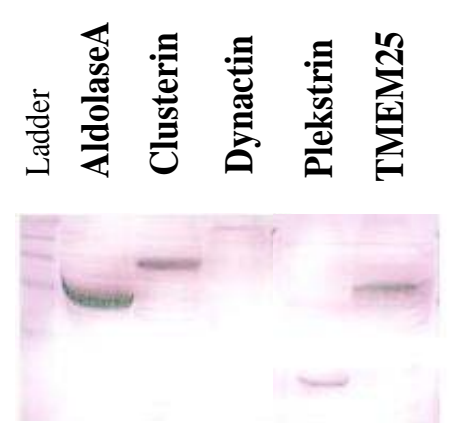

B.
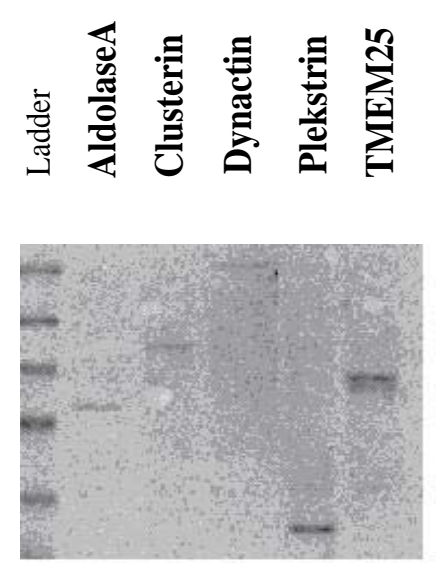

Fig. (2). Confirmation of Interaction with Selected Proteins in Mammalian Cells. The indicated fusion proteins were transfected along with EphB6 into mammalian cells. The samples were processed for detection of myc epitope (Panel A) or of EphB6 (Panel B) as described in the Materials and Methods section. Subsequenttly, aliquots of the lysates described in panel A were immunoprecipitated with an anti-EphB6 antibody (panel C) or a whole IgG antibody (panel D) as described in the Materials and Methods section and subjected to Western blotting with an anti-myc antibody. The control sample (Clusterin) in Panel D was immunoprecipitated with the EphB6 antibody to confirm the efficiency of the Western blot.

probing the blot with ant-EphB6 antibody. As shown in Fig. (2B), each transfectant is expressing abundant amounts of EphB6 protein. An aliquot $(200 \mu \mathrm{l})$ of cell lysate from each transfection was subjected to immunoprecipitation using an anti-EphB6 antibody (R\&D Systems) and analyzed for the presence of the myc-fusion protein. It is clear that all 5 fusion proteins were present in the immunoprecipitated samples (Fig. 2C). The presence of specific myc-fusion proteins in the immunoprecipitated samples suggests that they are interacting with the EphB6-fusion protein. Control immunoprecipitation reactions performed with whole goat IgG antibodies were insufficient to allow for detection of the fusion proteins (Fig. 2D). The lane marked control (Fig. 2D) represents the lysate from clusterin transfected cells that was immunoprecipitated with anti-EphB6 antibody and then probed with anti-Myc antibody. A positive signal in this lane demonstrates that the hybridization has worked efficiently. On the other hand, the absence of signal in other lanes demonstrates that the fusion proteins are not recognized by IgG antibody. Taken together, these results indicate specificity of coimmunoprecipitation and confirm the observed interactions.

After recognizing that these proteins are capable of interacting with EphB6 in HEK293T cells, TMEM25 was tested for its ability to interact with EphB6 in stable transfectants of MDA-MB-231 cells. Semi-quantitative RT-PCR and Western blotting experiments show that the levels of EphB6 mRNA and protein in MDA-MB-231 cells transfected with the full length EphB6 are significantly higher than the con- trol cells transefected with the empty vector (Fig. 3). The results presented in Fig. (4) indicate that TMEM25 is capable of interacting with EphB6 in MDA-MB-231 cells overexpressing EphB6. It warrants mention that the transfection efficiency of HEK293T cells is significantly higher than MDA-MB-231 cells as determined by transfection with green fluorescent protein (data not shown).

\section{DISCUSSION}

Many Eph receptors have been shown to play important roles in the progression of various types of cancer such as breast cancer, prostate cancer, colorectal cancer, neuroblastoma, melanoma and non-small cell lung cancer [12-17, 2123]. Specifically, the expression of EphB6 has been shown to be an important diagnostic marker in non-small cell lung carcinoma [13], melanoma [12], neuroblastoma [14-16] and breast cancer [17]. Although lacking intrinsic kinase activity, EphB6 can be phosphorylated upon dimerization with EphB1 [10]. Based on the high homology between EphB1 and other members of this family, it is likely that EphB6 gets phosphorylated after dimerization with other EphB receptors as well. Following phosphorylation, EphB6 can transduce signals in a phosphorylation-dependent manner [9-10, 24]. The phosphorylation of EphB6 following its heteromerization with other members of the EphB family could lead to a change in binding affinities for both the ephrin ligands as well as intracellular docking and/or signaling proteins as compared to unphosphorylated EphB6 homodimers [20]. 
Thus, unique expression patterns of the EphB family could alter the nature and magnitude of signaling through EphB6.

A.
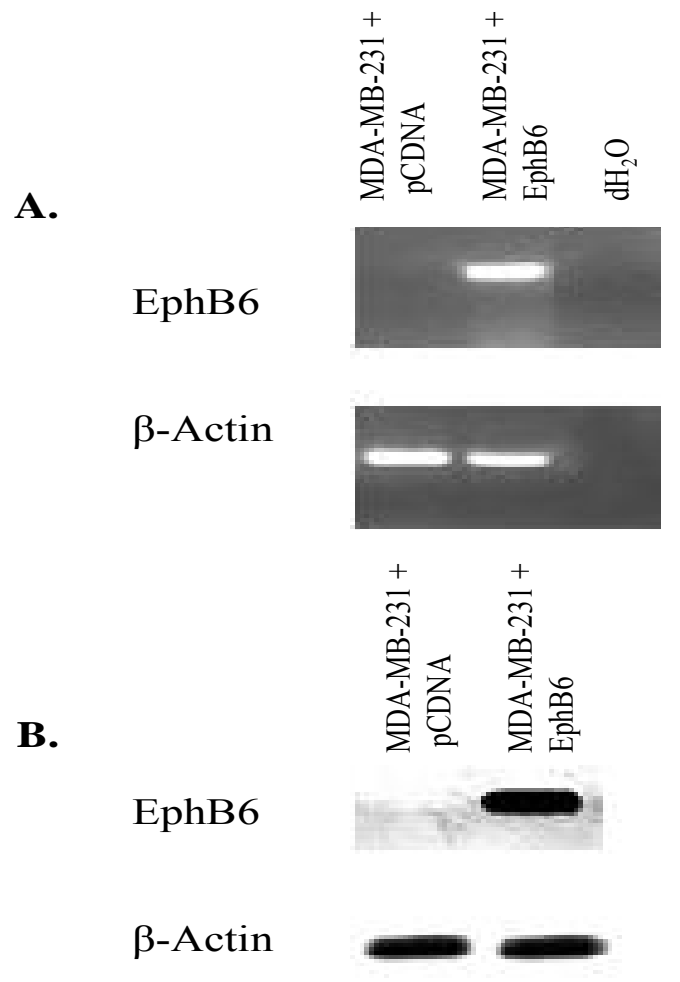

Fig. (3). Analysis of EphB6 Transcript and Protein Levels in MDA-MB-231 Clones. A. Amounts of EphB6 and $\beta$-actin transcripts among MDA-MB-231 clones were determined by RT-PCR as previously described. RT-PCR reactions were separated on a $1.5 \%$ agarose gel and photographed using Gel Doc (Bio-Rad) imaging software. B. Total cell lysates $(50 \mu \mathrm{g})$ were subjected to western blotting to determine EphB6 and $\beta$-actin protein levels as described previously. These experiments were performed three times and the results obtained were consistent.

Both ephrinB1 $[10,24]$ and ephrinB2 [11, 25] have been shown to bind and activate signaling through EphB6. In addition, EphB6 binds several intracellular proteins such as BCL6 [26], AF-6 [27], EphB1 [10], GRB2, CrkI, CrkII [9] and $\mathrm{Cbl}[9-10]$. Likewise, the protein tyrosine kinase $F y n$ has also been shown to constitutively bind to EphB6 [11]. The only phosphatase known to interact with EphB6 is the SH2domain-containing phosphatase SHP1 [28]. These proteins and other unidentified proteins are likely to regulate EphB6mediated signaling pathways.

The results presented in this paper demonstrate that aldolaseA, clusterin, dynactin, pleckstrin homology domaincontaining family B member 1 and TMEM 25 may also interact with EphB6. These interacting proteins were discovered using the cytoplasmic portion of EphB6 as bait in the yeast two-hybrid assay. The cytoplasmic region (405 amino acids) contains the tyrosine kinase domain, the sterile alpha motif (SAM) domain and the postsynaptic density, zonaoccludens (PDZ) domain [20]. An analysis of the domains contained within the newly identified interacting proteins described here is insufficient to explain the region of interaction with EphB6.

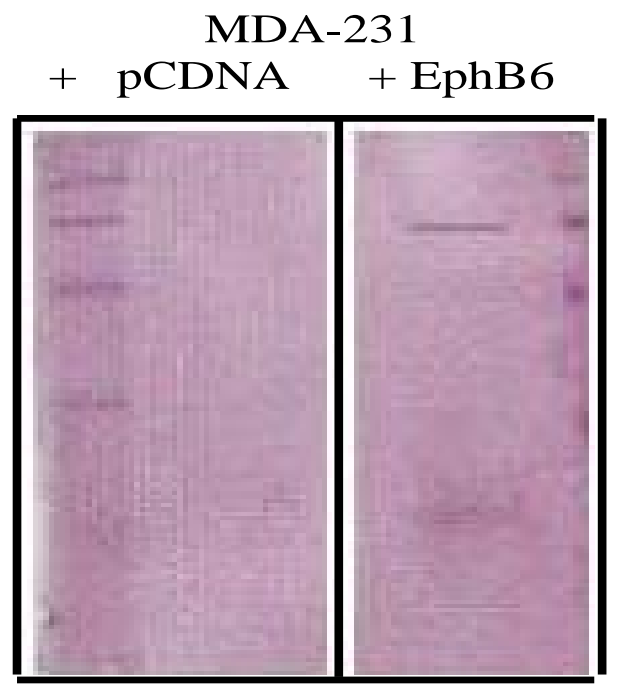

Fig. (4). EphB6 Interacts With TMEM25 When Overexpressed in MDA-MB-231 Cells. A TMEM25 fusion construct was transfected into stable MDA-MB-231 clones and tested for its ability to interact with EphB6 as described in the Materials and Methods section. Aliquots of protein lysates were immunoprecipitated with an anti-EphB6 antibody as previously described and subjected to Western blotting with an anti-myc antibody.

Mechanistically, these interacting proteins could impact the downstream signaling by shifting the balance of EphB6 homodimers relative to its heteromerization with other members of the EphB family. In addition to affecting the ratio of homomers and heteromers, the identified interacting proteins link EphB6 to additional signaling pathways. The pleckstrin domain-containing protein, for instance, is linked to signaling via phospholipids and its interaction with EphB6 may link EphB6 to signaling through lipid second messengers. Interestingly, several EphA receptors are known to signal through phosphatidylinositol 3-kinase in cultured cells $[29,30]$. TMEM 25 shares sequence homology with the immunoglobulin superfamily of proteins [31]. The members of this family of proteins have been implicated in cell adhesion and signaling via tyrosine kinase receptors [32-34]. Thus, the structure of TMEM25 is consistent with its potential ability to regulate Eph receptor-mediated signaling and adhesion/repulsion between cells.

The interaction of EphB6 with aldolaseA, clusterin and dynactin demands an alternative explanation. Although these cytosolic proteins are not known to actively participate in phosphorylation-dependent signaling events, some reports indirectly suggest their potential to influence EphB6mediated signaling. Dynactin is involved in vesicular trafficking via its regulation of the motor protein dynein [35], and is involved in regulating the movement of clathrincoated vesicles [36]. Although there is no direct experimental evidence for EphB6 involvement in clathrin-mediated trafficking, EphB2 and ephrinB1 have both been shown to be involved in this type of vesicular movement [37-39]. EphB2 has been shown to interact with synaptojanin 1, a phosphatidylinositol 5'-phosphatase that is involved in clathrin- 
mediated endocytosis [37]. EphrinB1 has also been shown to become internalized in clathrin-coated pits following its binding with soluble EphB1 receptors [38]. Significantly, ephrin B-mediated signal transduction is terminated by its endocytosis along with the receptor [39]. In this manner, dynactin may be involved in the removal of unphosphorylated EphB6 homomers from the membrane following ephrin stimulation.

The cellular functions of the two isoforms of clusterin (generated by alternative splicing) are complex. One of these two forms is a pro-apoptotic nuclear isoform and the other is a pro-survival secreted protein [40]. The secreted form of clusterin that was shown to interact with EphB6 is implicated in resistance to treatment in breast cancer and other cancers [40]. Clusterin has also been shown to bind to proteins to prevent stress related protein aggregation [41].

AldolaseA is an important enzyme of the glycolytic pathway [42]. No direct link can be established from the literature between aldolaseA and EphB6. It is possible that aldolaseA and EphB6 interact within caveolin-1 enriched membrane regions based on the observations that aldolase $\mathrm{A}$ [43] and Eph receptors [44, 45] are concentrated in these caveolae-like domains in tissue culture systems. Interestingly, several other EphB6 interacting proteins, including Fyn and Grb2 [9, 11, 45] are also known to cluster in these same regions.

We hypothesize that the interaction of EphB6 with the proteins identified in the yeast two-hybrid assay can interfere with its ability to form homodimers or heterodimers with other members of the EphB receptor family. Furthermore, such interactions can alter the phosphorylation status and biological activity of EphB6. The potential ability of EphB6 interactors to alter dimerization and phosphorylation status of EphB6 may lead to the development of novel therapeutic agents capable of modifying the state and activity of EphB6. It, however, remains to be confirmed if the interactions between EphB6 and the identified proteins exist at physiological concentration.

\section{REFERENCES}

[1] Manning, G.; Whyte, D.B.; Martinez, R.; Hunter, T.; Sudarsanam, $\mathrm{S}$. The protein kinase complement of the human genome. Science, 2002, 298, 1912-34

[2] Elowe, S.; Holland, S.J.; Kulkarni, S.; Pawson, T. Downregulation of the Ras-mitogen-activated protein kinase pathway by the EphB2 receptor tyrosine kinase is required for ephrin-induced neurite retraction. Mol. Cell Biol., 2001, 21, 7429-41.

[3] Holmberg, J.; Clarke, D.L.; Frisen, J. Regulation of repulsion versus adhesion by different splice forms of an Eph receptor. Nature, 2000, 408, 203-6.

[4] Kullander, K.; Klein, R. Mechanisms and functions of Eph and ephrin signalling. Nat. Rev. Mol. Cell Biol., 2002, 3, 475-86.

[5] Miao, H.; Wei, B.R.; Peehl, D.M.; Li, Q.; Alexandrou, T.; Schelling, J.R.; Rhim, J.S.; Sedor, J.R.; Burnett, E.; Wang, B. Activation of EphA receptor tyrosine kinase inhibits the Ras/MAPK pathway. Nat. Cell Biol., 2001, 3, 527-30.

[6] Pratt, R.L.; Kinch, M.S. Ligand binding up-regulates EphA2 messenger RNA through the mitogen-activated protein/extracellular signal-regulated kinase pathway. Mol. Cancer Res., 2003, 1, 10706.

[7] Zou, J.X.; Wang, B.; Kalo, M.S.; Zisch, A.H.; Pasquale, E.B.; Ruoslahti, E. An Eph receptor regulates integrin activity through RRas. Proc. Natl. Acad. Sci. USA, 1999, 96, 13813-8.

[8] Matsuoka, H.; Iwata, N.; Ito, M.; Shimoyama, M.; Nagata, A.; Chihara, K.; Takai, S.; Matsui, T. Expression of a kinase-defective
Eph-like receptor in the normal human brain. Biochem. Biophys. Res. Commun., 1997, 235, 487-92.

[9] Luo, H.; Wan, X.; Wu, Y.; Wu, J., Cross-linking of EphB6 resulting in signal transduction and apoptosis in Jurkat cells. J. Immunol., 2001, 167, 1362-70.

[10] Freywald, A.; Sharfe, N.; Roifman, C.M. The kinase-null EphB6 receptor undergoes transphosphorylation in a complex with EphB1. J. Biol. Chem., 2002, 277, 3823-8.

[11] Matsuoka, H.; Obama, H.; Kelly, M.L.; Matsui, T.; Nakamoto, M. Biphasic functions of the kinase-defective Ephb6 receptor in cell adhesion and migration. J. Biol. Chem., 2005, 280, 29355-63.

[12] Hafner, C.; Bataille, F.; Meyer, S.; Becker, B.; Roesch, A.; Landthaler, M.; Vogt, T. Loss of EphB6 expression in metastatic melanoma. Int. J. Oncol., 2003, 23, 1553-9.

[13] Muller-Tidow, C.; Diederichs, S.; Bulk, E.; Pohle, T.; Steffen, B.; Schwable, J.; Plewka, S.; Thomas, M.; Metzger, R.; Schneider, P.M.; Brandts, C.H.; Berdel, W.E.; Serve, H. Identification of metastasis-associated receptor tyrosine kinases in non-small cell lung cancer. Cancer Res., 2005, 65, 1778-82.

[14] Tang, X.X.; Zhao, H.; Robinson, M.E.; Cnaan, A.; London, W.; Cohn, S.L.; Cheung, N.K.; Brodeur, G.M.; Evans, A.E.; Ikegaki, N. Prognostic significance of EPHB6, EFNB2, and EFNB3 expressions in neuroblastoma. Med. Pediatr. Oncol., 2000, 35, 656-8.

[15] Tang, X.X.; Evans, A.E.; Zhao, H.; Cnaan, A.; Brodeur, G.M.; Ikegaki, N. Association among EPHB2, TrkA, and MYCN expression in low-stage neuroblastomas. Med. Pediatr. Oncol., 2001, 36, 80-2.

[16] Tang, X.X.; Robinson, M.E.; Riceberg, J.S.; Kim, D.Y.; Kung, B.; Titus, T.B.; Hayashi, S.; Flake, A.W.; Carpentieri, D.; Ikegaki, N. Favorable neuroblastoma genes and molecular therapeutics of neuroblastoma. Clin. Cancer Res., 2004, 10, 5837-44.

[17] Fox, B.P.; Kandpal, R.P. Invasiveness of breast carcinoma cells and transcript profile: Eph receptors and ephrin ligands as molecular markers of potential diagnostic and prognostic application. Biochem. Biophys. Res. Commun., 2004, 318, 882-92.

[18] Ding, B.; Lengyel, P. p204 protein is a novel modulator of ras activity. J. Biol. Chem., 2008, 283, 5831-48.

[19] Nagaraja, G.M.; Kandpal, R.P. Chromosome $13 q 12$ encoded Rho GTPase activating protein suppresses growth of breast carcinoma cells, and yeast two-hybrid screen shows its interaction with several proteins. Biochem. Biophys. Res. Commun., 2004, 313, 654-65.

[20] Himanen, J.P.; Nikolov, D.B. Eph signaling: a structural view. Trends. Neurosci., 2003, 26, 46-51.

[21] Alazzouzi, H.; Davalos, V.; Kokko, A.; Domingo, E.; Woerner, S.M.; Wilson, A.J.; Konrad, L.; Laiho, P.; Espin, E.; Armengol, M.; Imai, K.; Yamamoto, H.; Mariadason, J.M.; Gebert, J.F.; Aaltonen, L.A.; Schwartz, S. Jr.; Arango, D. Mechanisms of inactivation of the receptor tyrosine kinase EPHB2 in colorectal tumors. Cancer Res., 2005, 65, 10170-3.

[22] Batlle, E.; Bacani, J.; Begthel, H.; Jonkheer, S.; Gregorieff, A.; van de Born, M.; Malats, N.; Sancho, E.; Boon, E.; Pawson, T.; Gallinger, S.; Pals, S.; Clevers, H. EphB receptor activity suppresses colorectal cancer progression. Nature, 2005, 435, 1126-30.

[23] Surawska, H.; Ma, P.C.; Salgia, R. The role of ephrins and Eph receptors in cancer. Cytokine Growth Factor Rev., 2004, 15, 41933.

[24] Freywald, A.; Sharfe, N.; Rashotte, C.; Grunberger, T.; Roifman, C.M. The EphB6 receptor inhibits JNK activation in T lymphocytes and modulates $\mathrm{T}$ cell receptor-mediated responses. J. Biol. Chem., 2003, 278, 10150-6.

[25] Munthe, E.; Rian, E.; Holien, T.; Rasmussen, A.; Levy, F.O.; Aasheim, H. Ephrin-B2 is a candidate ligand for the Eph receptor, EphB6. FEBS. Lett., 2000, 466, 169-74.

[26] Miles, R.R.; Crockett, D.K.; Lim, M.S.; Elenitoba-Johnson, K.S. Analysis of BCL6-interacting proteins by tandem mass spectrometry. Mol. Cell Proteomics, 2005, 4, 1898-909.

[27] Buchert, M.; Schneider, S.; Meskenaite, V.; Adams, M.T.; Canaani, E.; Baechi, T.; Moelling, K.; Hovens, C.M. The junction-associated protein AF-6 interacts and clusters with specific Eph receptor tyrosine kinases at specialized sites of cell-cell contact in the brain. $J$. Cell Biol., 1999, 144, 361-71.

[28] Wimmer-Kleikamp, S.H.; Lackmann, M. Eph-modulated cell morphology, adhesion and motility in carcinogenesis. IUBMB Life, 2005, 57, 421-31.

[29] Fukushima, K.; Ueno, Y.; Inoue, J.; Kanno, N.; Shimosegawa, T., Filopodia formation via a specific Eph family member and PI3K in 
immortalized cholangiocytes. Am. J. Physiol. Gastrointest. Liver Physiol., 2006, 291, G812-9.

[30] Zhuang, G.; Hunter, S.; Hwang, Y.; Chen, J. Regulation of EphA2 receptor endocytosis by SHIP2 lipid phosphatase via phosphatidylinositol 3-Kinase-dependent Rac1 activation. J. Biol. Chem., 2007, 282, 2683-94.

[31] Katoh, M.; Katoh, M. Identification and characterization of human TMEM25 and mouse Tmem 25 genes in silico. Oncol. Rep., 2004, 12, 429-33.

[32] Meiri, K.F.; Saffell, J.L.; Walsh, F.S.; Doherty, P. Neurite outgrowth stimulated by neural cell adhesion molecules requires growth-associated protein-43 (GAP-43) function and is associated with GAP-43 phosphorylation in growth cones. J. Neurosci., 1998, 18, 10429-37.

[33] Niethammer, P.; Delling, M.; Sytnyk, V.; Dityatev, A.; Fukami, K.; Schachner, M. Cosignaling of NCAM via lipid rafts and the FGF receptor is required for neuritogenesis. J. Cell Biol., 2002, 157, $521-32$.

[34] Ronn, L.C.; Doherty, P.; Holm, A.; Berezin, V.; Bock, E. Neurite outgrowth induced by a synthetic peptide ligand of neural cell adhesion molecule requires fibroblast growth factor receptor activation. J. Neurochem., 2000, 75, 665-71.

[35] Schroer, T.A.; Dynactin. Annu. Rev. Cell Dev. Biol., 2004, 20, 75979.

[36] Zhao, Y.; Gaidarov, I.; Keen, J.H. Phosphoinositide 3-kinase C2alpha links clathrin to microtubule-dependent movement. $J$. Biol. Chem., 2007, 282, 1249-56.

[37] Irie, F.; Okuno, M.; Pasquale, E.B. Yamaguchi, Y., EphrinB-EphB signalling regulates clathrin-mediated endocytosis through tyrosine phosphorylation of synaptojanin 1. Nat. Cell Biol., 2005, 7, 501-9.
[38] Parker, M.; Roberts, R.; Enriquez, M.; Zhao, X.; Takahashi, T.; Pat Cerretti, D.; Daniel, T.; Chen, J. Reverse endocytosis of transmembrane ephrin-B ligands via a clathrin-mediated pathway. Biochem. Biophys. Res. Commun., 2004, 323, 17-23.

[39] Zimmer, M.; Palmer, A.; Kohler, J.; Klein, R. EphB-ephrinB bidirectional endocytosis terminates adhesion allowing contact mediated repulsion. Nat. Cell Biol., 2003, 5, 869-78.

[40] Shannan, B.; Seifert, M.; Leskov, K.; Willis, J.; Boothman, D.; Tilgen, W.; Reichrath, J. Challenge and promise: roles for clusterin in pathogenesis, progression and therapy of cancer. Cell Death Differ., 2006, 13, 12-9.

[41] Gleave, M.; Chi, K.N. Knock-down of the cytoprotective gene, clusterin, to enhance hormone and chemosensitivity in prostate and other cancers. Ann. N. Y. Acad. Sci., 2005, 1058, 1-15.

[42] Esposito, G.; Vitagliano, L.; Costanzo, P.; Borrelli, L.; Barone, R.; Pavone, L.; Izzo, P.; Zagari, A.; Salvatore, F. Human aldolase A natural mutants: relationship between flexibility of the C-terminal region and enzyme function. Biochem. J., 2004, 380, 51-6.

[43] Raikar, L.S.; Vallejo, J.; Lloyd, P.G.; Hardin, C.D. Overexpression of caveolin-1 results in increased plasma membrane targeting of glycolytic enzymes: the structural basis for a membrane associated metabolic compartment. J. Cell Biochem., 2006, 98, 861-71.

[44] Vihanto, M.M.; Vindis, C.; Djonov, V.; Cerretti, D.P.; Huynh-Do, U. Caveolin-1 is required for signaling and membrane targeting of EphB1 receptor tyrosine kinase. J. Cell Sci., 2006, 119, 2299-309.

[45] Wu, C.; Butz, S.; Ying, Y.; Anderson, R.G. Tyrosine kinase receptors concentrated in caveolae-like domains from neuronal plasma membrane. J. Biol. Chem., 1997, 272, 3554-9.

(C) Fox and Kandpal; Licensee Bentham Open.

This is an open access article licensed under the terms of the Creative Commons Attribution Non-Commercial License (http://creativecommons.org/licenses/ by-nc/3.0/) which permits unrestricted, non-commercial use, distribution and reproduction in any medium, provided the work is properly cited. 